\title{
Vector of Transplantation Incompatibility
}

National Cancer Institute

\section{Source}

National Cancer Institute. Vector of Transplantation Incompatibility. NCI Thesaurus.

Code C126297.

Refers to the direction of allorecog nition between donor and recipient. 